\title{
Engineering Hierarchical Nanostructures by Elastocapillary Self-Assembly
}

Michaël De Volder* and A. John Hart

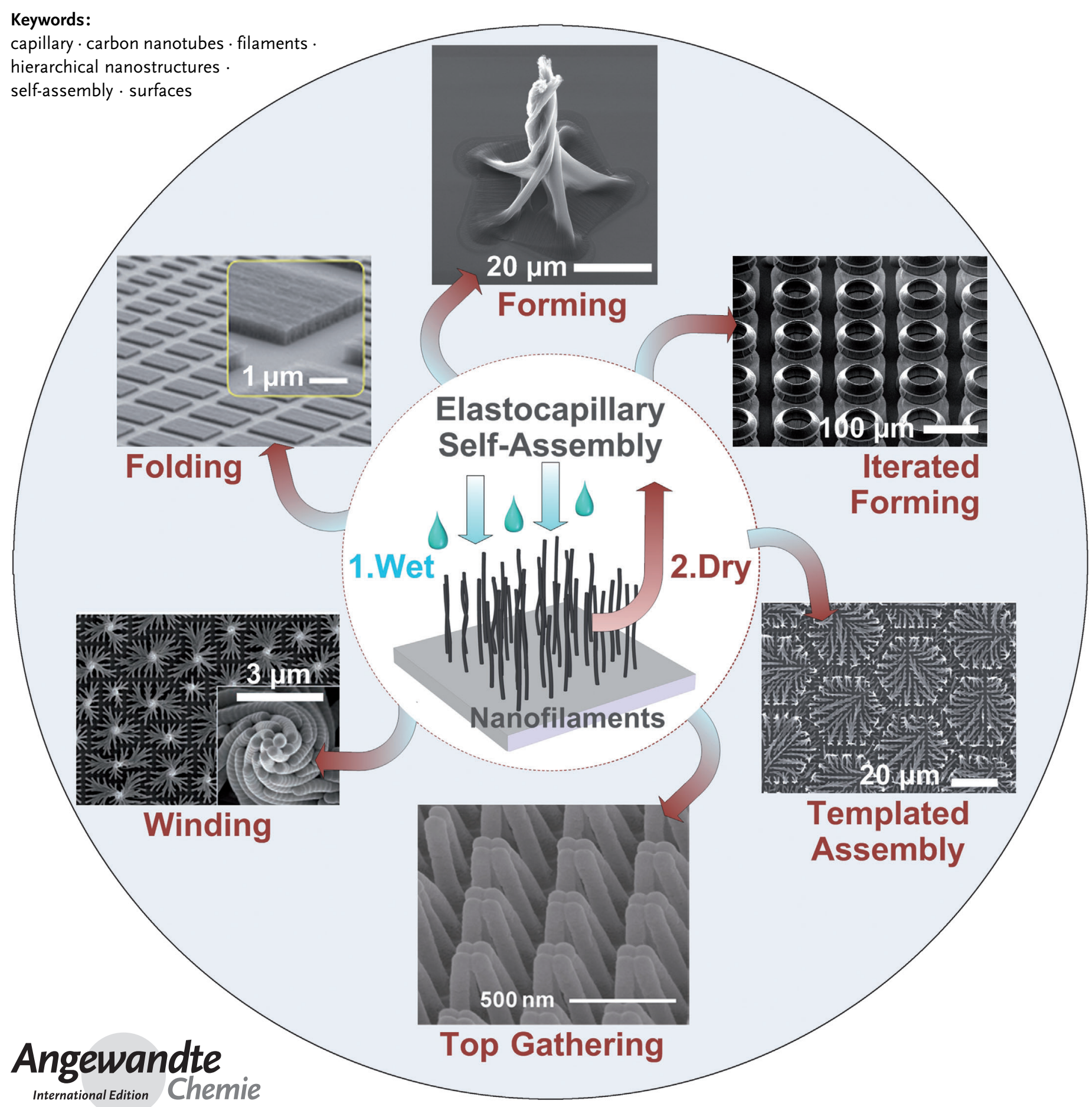


$\boldsymbol{S}_{\text {urfaces coated with nanoscale filaments such as silicon nanowires }}$ and carbon nanotubes are potentially compelling for high-performance battery and capacitor electrodes, photovoltaics, electrical interconnects, substrates for engineered cell growth, dry adhesives, and other smart materials. However, many of these applications require a wet environment or involve wet processing during their synthesis. The capillary forces introduced by these wet environments can lead to undesirable aggregation of nanoscale filaments, but control of capillary forces can enable manipulation of the filaments into discrete aggregates and novel hierarchical structures. Recent studies suggest that the elastocapillary self-assembly of nanofilaments can be a versatile and scalable means to build complex and robust surface architectures. To enable a wider understanding and use of elastocapillary self-assembly as a fabrication technology, we give an overview of the underlying fundamentals and classify typical implementations and surface designs for nanowires, nanotubes, and nanopillars made from a wide variety of materials. Finally, we discuss exemplary applications and future opportunities to realize new engineered surfaces by the elastocapillary self-assembly of nanofilaments.

\section{From the Contents}

\begin{tabular}{l} 
1. Introduction \\
$\begin{array}{l}\text { 2. Mechanism of Elastocapillary } \\
\text { Self-Assembly }\end{array}$ \\
$\begin{array}{l}\text { 3. Classification of Elastocapillary } \\
\text { Self-Assembly Methods }\end{array}$ \\
$\begin{array}{l}\text { 4. Classification of Surface } \\
\text { Designs Achieved by } \\
\text { Elastocapillary Self-Assembly }\end{array}$ \\
\begin{tabular}{l} 
5. Process Control \\
\hline $\begin{array}{l}\text { 6. Applications } \\
\text { 7. Summary and Outlook }\end{array}$
\end{tabular} \\
\hline
\end{tabular}

\section{Introduction}

Surfaces coated with high aspect ratio nanoscale filaments, such as polymer nanopillars, carbon nanotubes (CNTs), and semiconductor nanowires, offer unique properties that are distinct from the underlying substrate. These properties include tunable mechanical compliance and a large surface area for charge transport or electrochemical interaction. ${ }^{[1]}$ As a result, filamentary surfaces are highly attractive for applications including in supercapacitors, ${ }^{[2,3]}$ biomimetic adhesives, ${ }^{[4,5]}$ solar cells, ${ }^{[6,7]}$ superhydrophobic surfaces,${ }^{[8]}$ heat exchangers, ${ }^{[9]}$ DNA separation, ${ }^{[10]}$ biomedical diagnostics, ${ }^{[11]}$ materials for interfacing biological cells, ${ }^{[12,13]}$ and anisotropic surfaces. ${ }^{[14]}$

Importantly, many of these devices come in contact with a wet environment during formation of the filaments, during subsequent fabrication steps, ${ }^{[15]}$ or during use in the final application. ${ }^{[12]}$ This necessitates consideration of how capillary forces can influence the morphology of filaments on the surface. As a consequence of the compliance of high aspect ratio nanofilaments, as well as the relationship between capillary pressure and meniscus curvature, contact with liquids can easily manipulate these delicate structures. Furthermore, the relative strength of electrostatic and adhesive interactions (e.g. van der Waals forces) between nanostructures can alter their arrangement as the liquid evaporates. $^{[16]}$

While capillary aggregation was initially described as being detrimental to the processing of high-resolution polymer features made by lithography, ${ }^{[15]}$ advances in the controlled patterning of nanostructures have shown that capillary interactions can be used as a versatile and scalable fabrication step. For example, the wetting and drying of vertically aligned carbon nanotubes (CNTs) has been used to increase their packing density, ${ }^{[17-22]}$ to form periodic superstructures, ${ }^{[23-25]}$ to reorient thin films into horizontal sheets and multidirectional circuits, ${ }^{[26-30]}$ and to fabricate intricate $3 \mathrm{D}$ microarchitectures. ${ }^{[28,31-35]}$ Similar principles have been used to manipulate nanofilaments made out of $\mathrm{Si}^{\left[{ }^{[36-39]}\right.} \mathrm{Au}^{[40]} \mathrm{ZnO},{ }^{[41]} \mathrm{Cu},{ }^{[38]}$ hydrogels, ${ }^{[1,42]}$ epoxy, ${ }^{[1,16]}$ PMMA, ${ }^{[12,43,44]}$ PET, ${ }^{[45]}$ cyclic olefin copolymers, ${ }^{[12]}$ polyurethane, ${ }^{[1]}$ PDMS,${ }^{[46]}$ various photoresists, ${ }^{[15,47,48]}$ and even biological fibers. ${ }^{[49]}$

This Review addresses the methods, mechanisms, and applications of the elastocapillary self-assembly of vertical nanofilaments on substrates. The goals of this Review are to: 1) Explain and classify the methods currently used to perform the elastocapillary self-assembly of nanofilaments; 2) provide a comprehensive overview of how these methods can be applied to create disordered and ordered 2D and 3D structures; 3) review the physical models and present practical guidelines to design and optimize the elastocapillary selfassembly process; and 4) identify current and future applications in nano/microfabrication and surface engineering. Besides the current state of the art, this Review also includes unpublished observations from our own research on capillary self-assembly. We also note that capillary self-assembly has been used to manipulate discrete particles and patterned thin

\section{[*] Dr. M. De Volder}

imec and Department of Mechanical Engineering

KULeuven, Heverlee (Belgium)

E-mail: michael.devolder@imec.be

Dr. M. De Volder

School of Engineering and Applied Sciences

Harvard University, Cambridge, Ma (USA)

Prof. A. J. Hart

Department of Mechanical Engineering

University of Michigan, Ann Arbor, MI (USA) 
a)
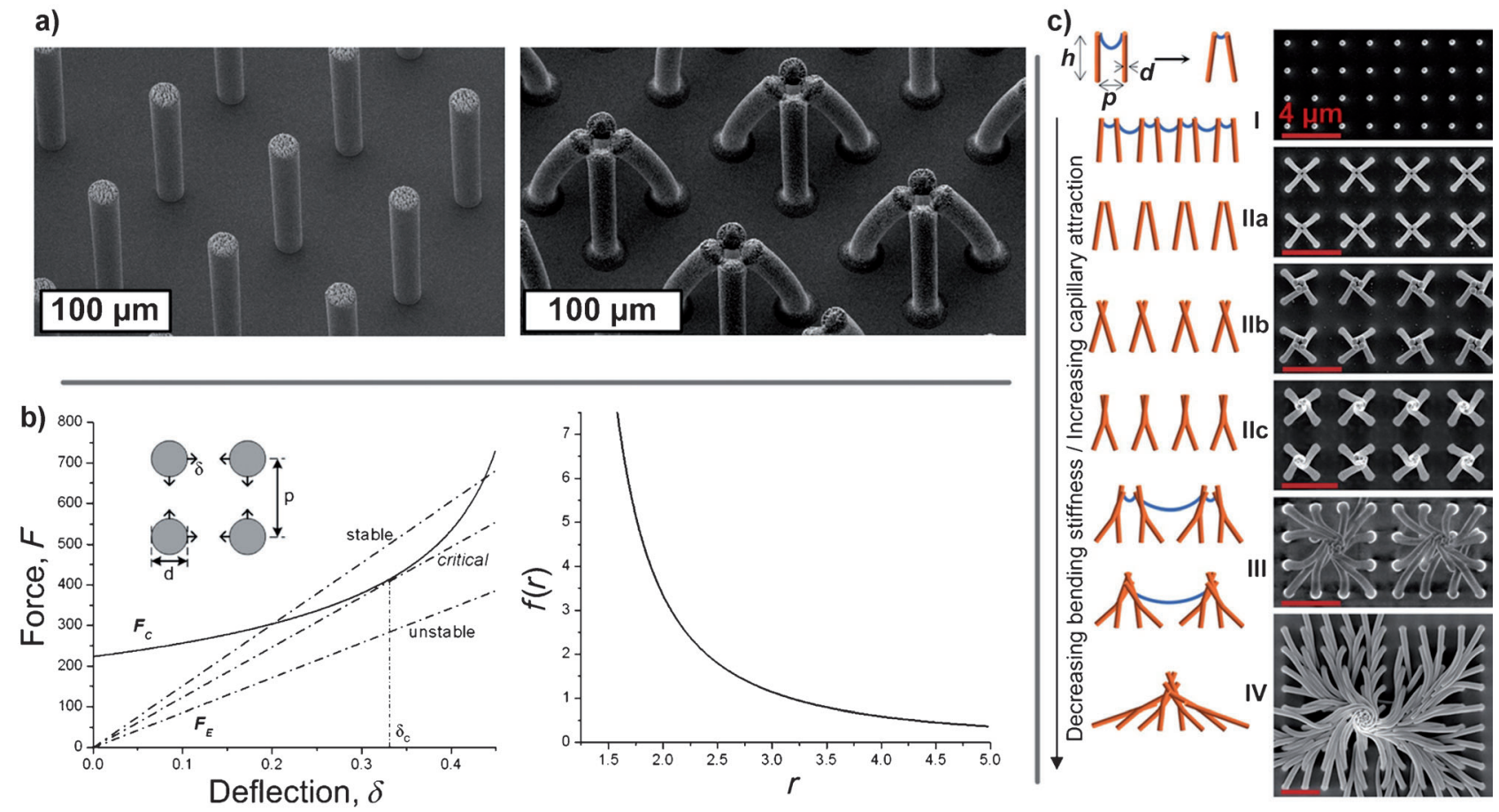

Figure 1. a) Epoxy micropillars before (left) and after (right) aggregating in groups of four through capillary self-assembly. b) Model of the aggregation of pillars in groups of four pillars [Eq. (1)]. The left graph shows the capillary interaction force $F_{\mathrm{C}}$ and the elastic restoring force $F_{\mathrm{E}}$. The right graph shows $f$ as a function of $r=P / d$. Reprinted from Ref. [1] with permission. Copyright 2010 American Chemical Society. c) Evolution of the nanofilament cluster from groups of four to complex helical aggregates as the length of the filaments increases (scale bar $4 \mu \mathrm{m}$ ). Reprinted from Ref. [55] with permission. Copyright AAAS.

films (including "micro-origami" structures). This topic is beyond the scope of this Review, and for these topics, we refer the reader to other reviews. ${ }^{[0-54]}$

\section{Mechanism of Elastocapillary Self-Assembly}

We define elastocapillary self-assembly (sometimes called elastocapillary aggregation) as the mechanical deformation and change in the arrangement of a population of filaments when influenced by capillary forces during wetting or drying of a liquid. As a meniscus recedes, it exerts a lateral force on nearby filaments. If these capillary forces are stronger than the elastic restoring forces for the deformed filaments, they will aggregate and come into contact with one another. If the surface forces between the contacting filaments then exceed

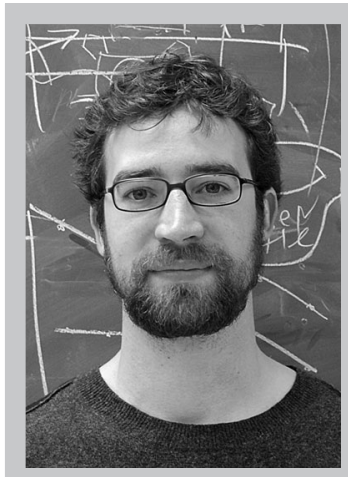

Michaël De Volder completed his PhD in the field of microsystems technology at the KULeuven, Belgium, and in part at the Tokyo Institute of Technology, Japan. Subsequently, he carried out postdoctoral research in the field of carbon nanotubes and capillary self-assembly at the University of Michigan, MIT, and Harvard Univeristy in the USA and at imec and KULeuven in Belgium. He is a Laureate of the Royal Academy of Science and Fine Arts, Belgium, as well as the recipient of several prestigious academic and industrial awards. the elastic restoring forces, the aggregated configuration can be stable after the liquid evaporates. A basic example is shown in Figure $1 \mathrm{a}$, where a square-grid array of epoxy micropillars aggregate in clusters of four "top-gathering" pillars. In this case, the surface forces between the pillars in contact (at the tips) are stronger than the elastic forces as a result of deflection of the pillars, and the clusters are stable after the liquid recedes.

Analytical models have been shown to predict the aggregated state after drying. For example, Chandra and Yang $^{[1]}$ observed that pillars assemble as soon as their tips pierce the liquid surface. By equating the elastic and capillary forces, they derived the minimal required elastic modulus $E_{\text {crit }}$ needed to resist elastocapillary aggregation, ${ }^{[1]}$ that is, for surface tension to hold the pillars together. By using an array of regularly spaced nanofilaments as a model system (Figure $1 \mathrm{a}, \mathrm{b})$, Equation (1) can be deduced: $:^{[1]}$

$E_{\text {crit }}=\frac{32 \sqrt{2} \gamma \cos ^{2} \theta h^{3}}{3 d^{4}} f(r)$

Here, $\theta$ is the contact angle, $\gamma$ is the surface tension, $h$ the pillar height, $d$ is the pillar diameter, and $f(r)$ is a function of the diameter and spacing of the pillars shown in Figure $1 \mathrm{~b}$.

This approach has been extended to understand the clustering behavior of aggregates containing larger numbers of nanofilaments. ${ }^{[1]}$ When the filaments bend inward toward one another, the spacing among the tips decreases, thereby resulting in a decrease in the capillary energy, and an increase 
in the elastic bending energy. When a group (cluster) of filaments gathers toward a common point, the outer filaments must bend more than the inner ones. Thus, the bending energy per filament increases with cluster size. This can only be accommodated by the surface tension for flexible (e.g. long) filaments. The resulting cluster size $N_{c}$ is then given as Equation (2): ${ }^{[1]}$

$N_{c}=\frac{273 \cos ^{2} \theta \gamma h^{3}}{E d^{2} w^{2}}$

Here, $\theta$ is the contact angle, $\gamma$ is the surface tension, $h$ is the pillar height, $E$ is the Young's Modulus, $d$ is the pillar diameter, and $w$ is the space between neighboring pillars (assuming a square grid, as in Figure 1B).

After the liquid has evaporated and the nanofilaments are dry, adhesion (e.g. van der Waals interactions) will determine the stability of the aggregated state. Indeed, in a dry state, the filament-filament interactions have to balance the elastic energy, and as such, they play a key role in the final geometry of the aggregates. ${ }^{[16,46,56,57]}$ In other words, the balance between capillary attraction and elastic restoration determines the maximum number of pillars that can aggregate in one bundle, while the balance between adhesion and elasticity determines how many nanofilaments will be preserved in the final structure after drying. ${ }^{[16,56]}$ Later on, we will discuss how filaments can aggregate in complex helical bristles and other $3 \mathrm{D}$ geometries, as shown in Figure $1 \mathrm{C}$. $^{[43,55,56]}$

An important assumption of these models is that the filaments are isolated, parallel, and rigidly clamped to the substrate. These assumptions apply to a wide variety of engineered nanofilaments because most fabrication methods result in arrays of relatively straight pillars that are spaced uniformly. Such methods include direct electron-beam writing of polymer nanowires in a photoresist, ${ }^{[43]}$ replica molding of silicon masters to produce polymer pillar arrays, ${ }^{[58]}$ anisotropic etching of silicon posts, ${ }^{[59,60]}$ and growth of nanowires and nanotubes by chemical vapor deposition (CVD). ${ }^{[61-63]}$ However, the high flexibility of very small diameter filaments such as carbon nanotubes (CNTs) leads to waviness and entanglement in their geometry, which makes the aggregation behavior also depend on the organization and overall shape of the population of filaments. In the following we will also refer to these large populations of tangled nanofilaments as "forests". Further caveats will be described later on; for the moment, the important message is that capillary self-assembly is influenced by the liquid $(\gamma, \theta)$, the pillar material $(E$, surface chemistry), and the geometry $(d, h, w)$.

\section{Classification of Elastocapillary Self-Assembly Methods}

The method by which the filaments are exposed to the liquid (i.e. the wetting and drying steps) influences the final assembly. Here, we discuss the main assembly methods that have been developed to date. These methods are summarized in Figure 2.
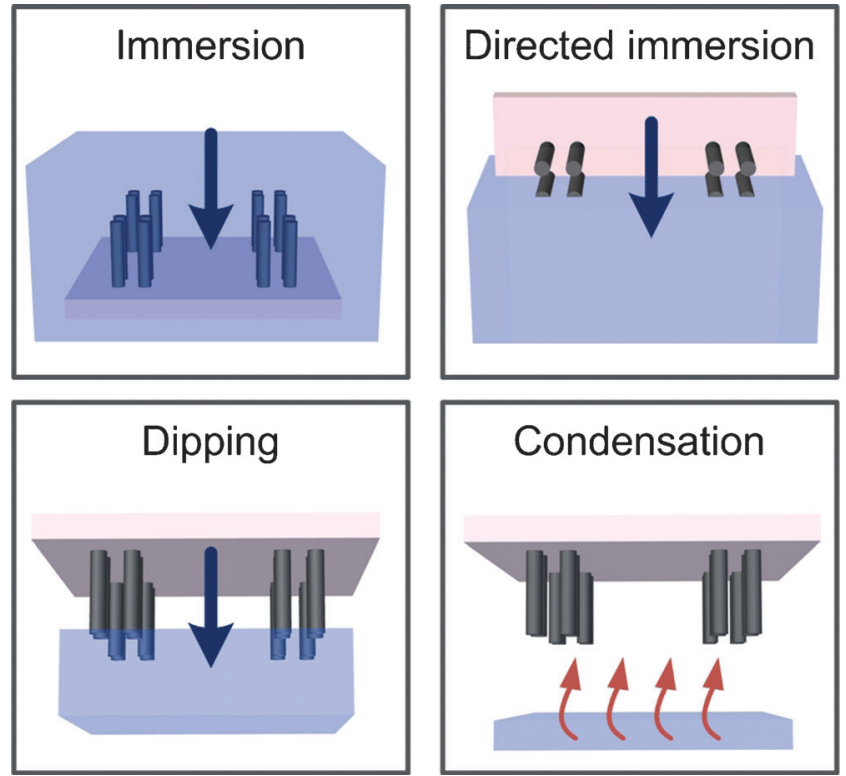

Figure 2. Overview of wetting methods used for elastocapillary selfassembly.

Immersion: The simplest and most common method of capillary self-assembly is to immerse the substrate in the beaker containing a wetting liquid, ${ }^{[3,13,23,43,55,64-67]}$ which is typically an organic solvent. The substrate is then removed from the beaker and the liquid is evaporated under ambient conditions. Here, the orientation of the substrate is not specified to be important, and is generally assumed to be parallel to the liquid surface. This process can readily be applied to segregate various types of nanorods and nanotubes.

Directed Immersion: In the case of directed immersion, the substrate is oriented in a specific direction with respect to the liquid surface, so as to control the direction of capillary forces that the structures experience as they contact the liquid surface. Specifically, this method was developed to change the orientation of nanofilaments as they are drawn from the liquid, ${ }^{[27]}$ thereby causing the filaments to change from a vertical to a horizontal alignment (parallel to the substrate). ${ }^{[27,29]}$ An example is the folding of vertical CNT microstructures to form horizontally aligned films. ${ }^{[27,29]}$

Dipping: Variations of the immersion process were developed to enable more localized and uniform control of the capillary forces applied to the nanofilaments. In the dipping method, the substrate is inverted parallel to the liquid surface, and then lowered until the tips of the nanofilaments touch the liquid surface. The liquid is then drawn into the spaces between the nanofilaments through capillary action. In principle, as the liquid is absorbed locally, ${ }^{[17,65,68]}$ this method should aid the uniformity of top-gathering assembly, as described in the next section.

Condensation: More delicate control over the introduction of the wetting liquid is achieved by condensing the liquid onto the substrate and the nanofilaments. This is especially useful for processing fragile structures that may be damaged (e.g. delaminated, aggregated, or undesirably reoriented) due to the capillary forces exerted by the surface of the liquid 
during immersion or dipping. In this method, the substrate is inverted over a tall beaker containing a small amount of heated liquid, such as acetone, as shown in Figure $2 .{ }^{[35]}$ As the substrate remains at a lower temperature than the liquid, the vapor condenses on the substrate, thereby enabling a selfdirected capillary rise into individual groups of structures on the substrate. ${ }^{[19,24,69]}$

Finally, we note that some researchers have combined these wetting methods with a mechanical pretreatment to first direct the rearrangement of vertical nanofilaments, for example by shearing ${ }^{[20]}$ or rolling, ${ }^{[70]}$ before immersion and drying. Another method for delicate liquid delivery uses a bubble to contact the tips of patterned CNT forests, thereby causing local densification of the upper portions of the CNT forests. ${ }^{[69]}$

\section{Classification of Surface Designs Achieved by Elastocapillary Self-Assembly}

To develop design rules that are based on theory as well as insight and intuition, it is useful to classify the geometries that can be achieved by elastocapillary self-assembly. For each classification, we provide visual examples of different numbers of pillars, $N=1-10, N=1-100$, and $N>100$. In the last case, forests of intertwined filaments such as CNTs are mostly used. These are summarized in Figure 3, and discussed in detail as follows.

Top Gathering: One of the first in-depth studies of the capillary self-assembly of nanofilaments was reported by Tanaka et al. ${ }^{[15]}$ in 1993, who used patterned polymer nanopillars with a diameter of $200 \mathrm{~nm}$. They observed the formation of top-gathering, "zipping" pyramidal huts after rinsing and drying of the developing liquid. Typical examples of top-gathering pillars with an increasing number per assembly are show in Figure 4. As described in the study by Tanaka et al., the self-assembly process is greatly influenced by the aspect ratio and spacing of the nanofilaments [as explained by Eqs. (1) and (2)]. While the nanofilaments could ideally aggregate into perfect closely packed clusters, the bending stiffness of the filaments, as well as variations in the initial spacing, waviness, or even entwining of the nanofilament, typically prevents a perfect packing from being achieved.$^{[3,19,74]}$ For example, top-gathering densification of CNT forests enhanced their cross-sectional density 5- to 25fold by a dip-coating method, ${ }^{[68]}$ by 20 -fold for delaminated SWNT forests of $2.8 \mathrm{~nm}$ diameter, ${ }^{[3]}$ and by 30 -fold for plasma-treated MWNT forests (Figure 4e) ${ }^{[19]}$ However, this is typically no more than $50 \%$ of the ideal density of hexagonally packed CNTs.

Winding: Kang et al. described how interplay between the stiffness, geometry, and chemistry of nanofilaments can cause aggregation into chiral (twisted) structures (Figure 5), rather than top-gathering pyramids (Figure 4). ${ }^{[56]}$ The transition from a top-gathering assembly to a chiral winding assembly depends on a delicate balance between adhesion, elasticity, and capillarity. ${ }^{[56]}$ For example, polymer nanopillars with a strong adhesion to one another formed huts, while nanopillars with weaker adhesion that permitted slip formed chiral aggregates. ${ }^{[56]}$ After the pillars contact one another at their tips, chirality arises when the contact point slips and the pillars twist around one another. Enhancing the adhesion by plasma treatment of epoxy nanofilaments prevented slip, and therefore prohibited winding. Similarly, large stiff pillars also prevent chiral winding because of the high elastic restoring force. Incidentally, we observed slight winding after the capillary self-assembly of high aspect ratio CNT micropillars. This is a hierarchical process where each individual pillar aggregates (as in Figure 4e), and at the same time, groups of pillars are crowded into the winding structures shown in Figure $5 \mathrm{f}$.

Folding: While top-gathering and winding is caused by capillary forces among filaments (i.e. bridging menisci), capillary forces between the filaments and the substrate can cause the filaments to collapse to the substrate. Therefore, wetting and drying can also enable controlled folding of filaments and thin vertical structures. For the model system of an isolated filament, reorientation occurs when the vertical force exerted by the wetting meniscus exceeds the force necessary to buckle the structure to the substrate. Reorientation may also occur as the meniscus bends the structure toward the substrate during evaporation. In any case, "zipping" of the meniscus between the structure and substrate during evaporation promotes the stability of the folded configuration. ${ }^{[26]}$ This principle has been applied to fold rows of asymmetric PMMA beams with a width of $10 \mathrm{~nm}$ (Figure 6a,b,d) ${ }^{[4]}$ as well as PMMA nanosheets (Figure 6c) ${ }^{[72]}$ CNT forest microwalls were folded by directed immersion (Figure 6e), ${ }^{[27,29]}$ thereby resulting in horizontally aligned (HA-)CNT films with a packing fraction of $42 \%$. Here, all the CNTs collapse in the same direction, guided by the immersion direction. Later, it was found that the direction of CNT folding could be specified by engineering asymmetry into the CNT forest cross-section, which enabled the fabrication of multidirectional HA-CNT patterns over large areas (Figure $6 \mathrm{f}){ }^{[26]}$

Foaming: When films of closely spaced and flexible nanopillars are exposed to a wetting liquid, large aggregates form which resemble a two-dimensional open-cell foam. This behavior has largely been studied for CNT forests, ${ }^{[19,23,24,33,64,76]}$ as well as arrays of $\mathrm{Si}^{[36,37,66]} \mathrm{Cu},{ }^{[66]}$ and $\mathrm{ZnO}^{[41]}$ nanowires. In the case of CNT forests, the contraction of the forest during liquid evaporation, in competition with the stiffness of the CNTs and their adhesion to the substrate, causes the formation of randomly distributed voids within the final assembly. For CNTs, Chakrapani et al. ${ }^{[23]}$ found that the average void size varies linearly with the length (height) of the CNTs in the forest.

For model systems, the "phase boundary" between the formation of voids and the formation of single aggregates can be predicted analytically, and shows good agreement with experimental results. ${ }^{[19]}$ Numerical models based on networks of springs also predict the distribution and geometry of the voids surprisingly well. ${ }^{[76]}$

Furthermore, while nonpatterned CNT forests result in randomly distributed voids, the arrangement of voids can be programmed by patterning holes in the forest before selfassembly. This is done by patterning the catalyst prior to CNT 

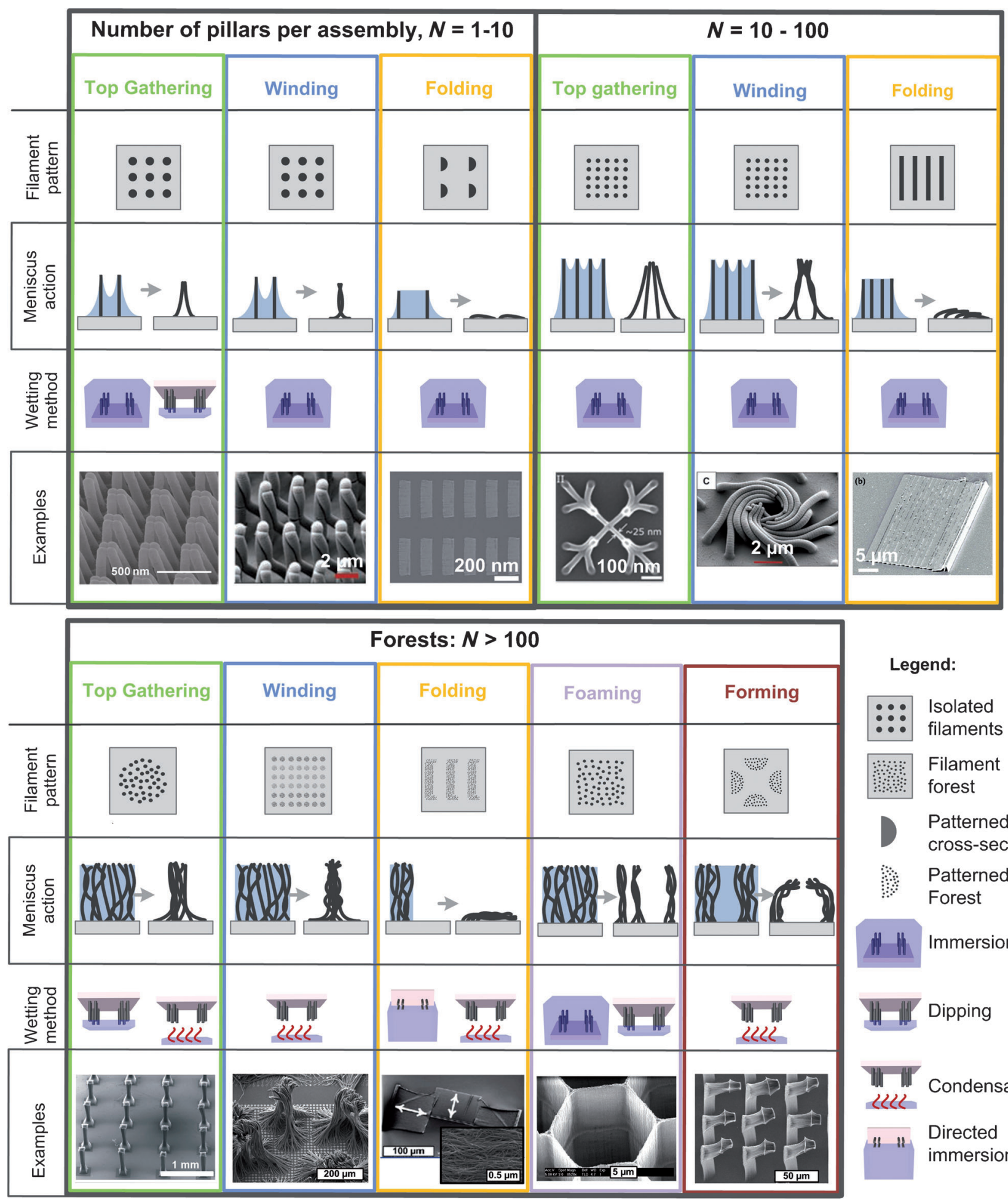

Legend:

$\because:$ Isolated

$\because:$ filaments

Filament

forest

Patterned cross-section

Patterned
Forest

II. If Immersion

III. III Dipping

«U Condensation
"II II $\begin{aligned} & \text { Directed } \\ & \text { immersion }\end{aligned}$

Figure 3. Classification of surface designs achieved by elastocapillary self-assembly according to the number of nanofilaments per aggregate and the principal assembly motion: top-gathering, winding, folding, forming, and foaming. From left to right, reprinted from Ref. [71] with permission (Copyright 2011 American Chemical Society); from Ref. [55] with permission (Copyright AAAS); from Ref. [44] with permission (Copyright 2011 Wiley); from Ref. [43] with permission (Copyright 2010 American Chemical Society); from Ref. [72] with permission (Copyright 2011 IOP); from Ref. [73] with permission (Copyright 2011 IEEE); from Ref. [26] with permission (Copyright 2011 American Chemical Society).

growth or by etching the CNT forest after growth (e.g. by using laser ablation). The holes initiate void formation and, therefore, enable the generation of highly uniform periodic arrangements of cells over large areas. Depending on the hole pattern, hexagonal ("honeycomb"), square, or other lattices are achieved, as shown in Figure $7 \mathrm{c}, \mathrm{d}$ and in the literature. ${ }^{[23,24,33,64]}$ Moreover, CNT foams are mechanically robust, and can be directly peeled from the substrate or separated 

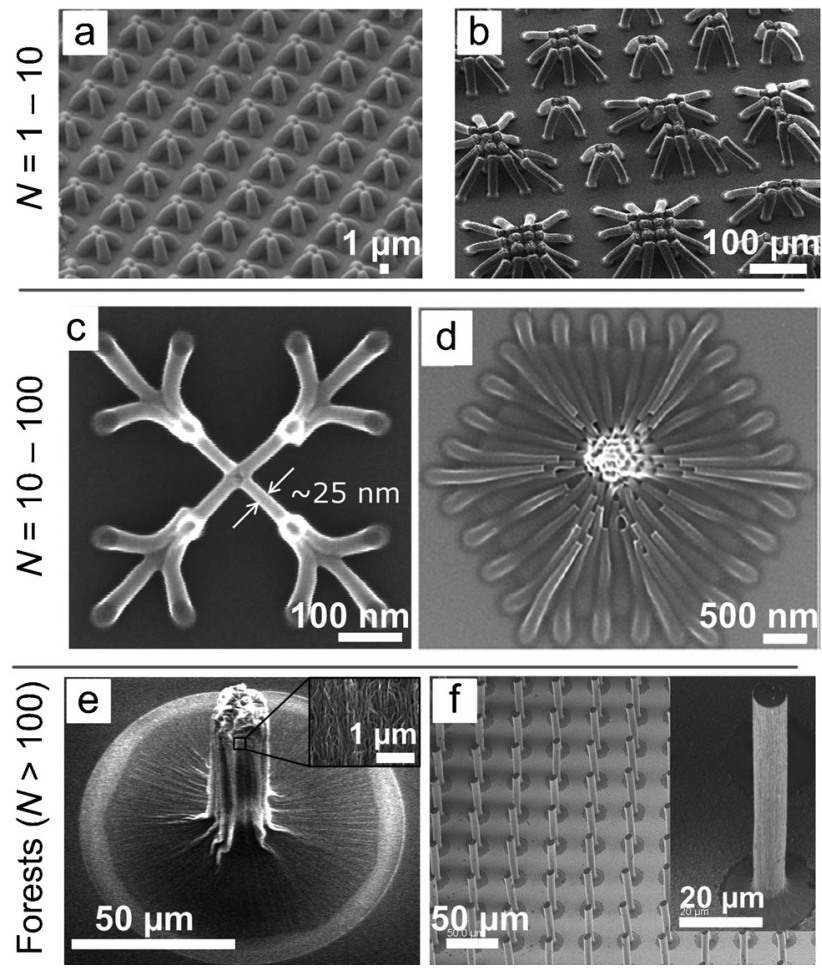

Figure 4. Examples of top-gathering pillars made by capillary selfassembly. a) Photoresist pillars fabricated by multibeam lithography. Reprinted from Ref. [75] with permission. Copyright 2009, American Institute of Physics. b) SU8-epoxy pillars after capillary self-assembly and subsequent pyrolysis, similar to in Ref. [59]. c,d) Arrays of polymer nanopillars made by electron-beam lithography, then capillary selfassembled into clusters. Reprinted from Ref. [43] with permission. Copyright 2010 American Chemical Society. e,f) CNT forest micropillars that aggregate by capillary self-assembly. Reprinted from Ref. [19] with permission. Copyright 2011 IOP and 2006 Macmillan. ${ }^{[3]}$

more gently by immersion in dilute aqueous hydrofluoric acid.

Forming: Within the dimensional bounds that form discrete solid aggregates upon self-assembly (i.e. no foaming regime), it was recently found that a variety of intricate $3 \mathrm{D}$ filament aggregates can be made by considering the global distribution of capillary forces during wetting and drying. ${ }^{[24]}$ For example, it was found by starting from CNT forest microstructures grown from a lithographically patterned catalyst that condensation densification transforms thinwalled cylinders into sloped microwells upon aggregation (Figure 7e), and causes semicylindrical posts to deflect laterally (Figure $7 \mathrm{f}$ ). More-complex geometries can be programmed to form helices with deterministic handedness (Figure $7 \mathrm{~g}$ ). ${ }^{[32]}$ In these examples, the initial geometry of the CNT forest, in combination with the density and alignment of the CNTs within the forest, defines the final geometry of the structures. ${ }^{[24,78]}$

Moreover, the capillary "forming" of CNTs can be iterated to create complex corrugated microstructures, including symmetric (see Figure $7 \mathrm{~h}$ ) and asymmetric shapes (see "forming" in Figure 3). ${ }^{[33,79]}$ Analytical models ${ }^{[78]}$ and finite element models ${ }^{[73]}$ can predict the basic shape transformations that occur by capillary forming. Therefore, along with
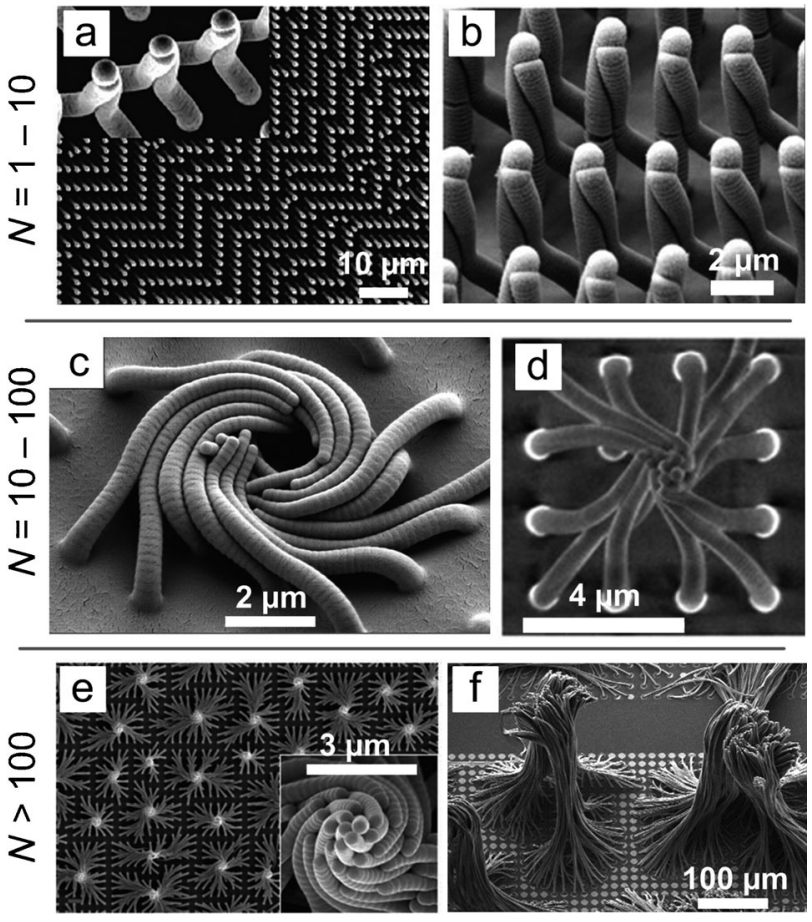

Figure 5. Winding aggregates of nanofilaments as a function of the number of pillars per aggregate. a-e) Winding of polymeric filaments. a) Reprinted from Ref. [56] with permission. Copyright 2010 American Chemical Society. b-e) Reprinted from Ref. [55] with permission. Copyright AAAS. f) Generation of carbon nanotube forests.

the expected improvements in CNT growth and characterization, there is a credible path toward using capillary forming to build large-area 3D microstructured surfaces using CNTs.

\section{Process Control}

Beyond establishing the filament material, geometry, and organization, other fabrication steps and process modifications have been shown to influence the self-assembly process, and to enable additional functionality of the final surfaces. This section provides a brief overview of these methods.

Surface Chemistry: The surface chemistry of the filaments influences the strength and stability of their interactions during self-assembly. This was investigated by coating epoxy nanopillars with a thin gold layer followed by attachment of alkanethiol self-assembled monolayers (SAMs) to the surface (Figure $8 \mathrm{a}$ ). It was found that in the case of short-chain molecules that the percentage of stable clusters scales with the strengths of the chemical bonds expected to form by the respective functional groups. Furthermore, SAMs with longer carbon chains produce more-ordered monolayers and, therefore, usually more stable clusters. ${ }^{[16]}$ These surface interactions enable the reversibility of the assembly to be controlled; for example, reducing the interfilament adhesion allows the filaments to spring-back as the liquid evaporates. ${ }^{[16]}$

Liquid and Evaporation Method: In principle, any liquid that wets the filaments and the substrate can be used for elastocapillary self-assembly. ${ }^{[15]}$ However, the influence of the liquid properties, such as, for example, the surface tension, ${ }^{[19]}$ 

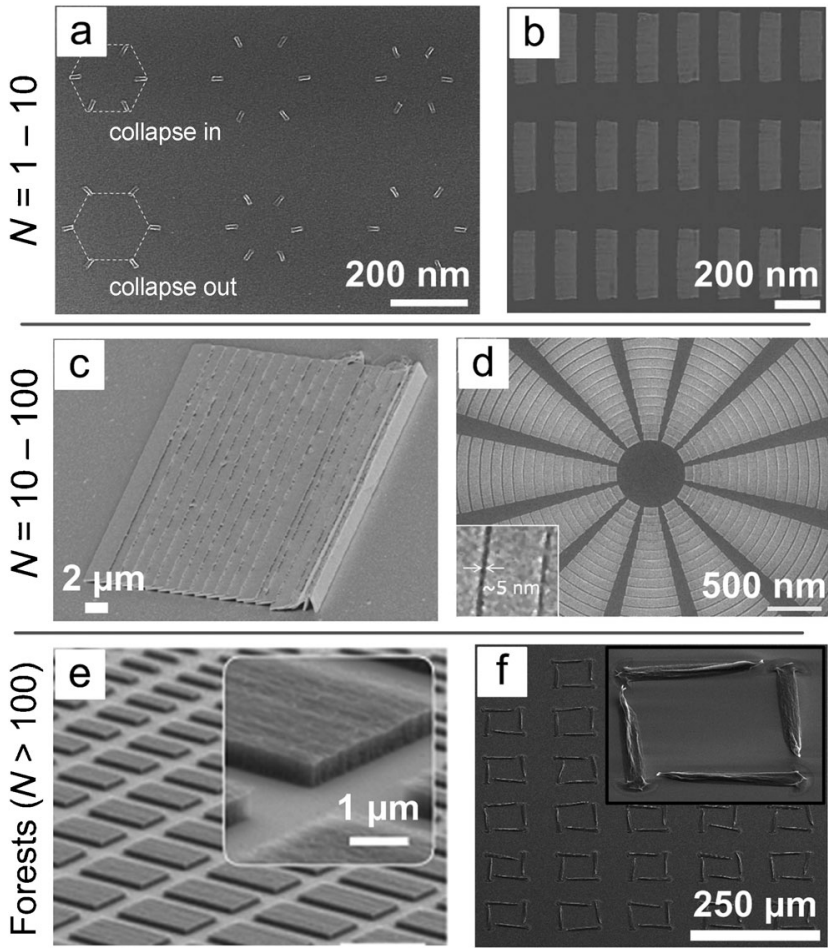

Figure 6. Elastocapillary folding of nanopillars as a function of the number of pillars per aggregate. a,b,d) Electron-beam photoresist lines folded by immersion densification. Reprinted from Ref. [44] with permission. Copyright 2011 Wiley. c) PMMA sheets folded by immersion densification. Reprinted from Ref. [72] with permission. Copyright 2011 IOP. e) CNT forest folded by directed immersion. Reprinted from Ref. [27]. Copyright 2008 Macmillan. f) Multidirectional CNT forests folded by the condensation method. Reprinted from Ref. [26] with permission. Copyright 2011 American Chemical Society.

viscosity, and the dynamics of the wetting and drying (e.g. evaporation rate) remains under study. For example, Chakrapani et al. reported the densification of $\mathrm{CNT}$ forests into cellular foams by using acetone, toluene, dimethylformamide, tetrahydrofuran, and methanol. ${ }^{[23]}$ Futuba et al. reported CNT densification by using water, alcohols, acetone, hexane, cyclohexane, dimethylformamide, liquid nitrogen, dioctyl ether, oleic acid, and machine oils. ${ }^{[3]}$

In the case of polymer nanopillar arrays, it was found that controlling the evaporation rate enables better uniformity of the process. $^{[16]}$ Figure $8 \mathrm{~b}$ shows the boundaries over which top-gathering pillars aggregated uniformly. Moreover, some researchers claim that self-assembly happens only as the liquid withdraws from the nanowires during evaporation, while others claim aggregation happens both during wetting and drying. The former claim has been verified for various material systems, by using freeze drying ${ }^{[12,23]}$ and performing the AFM analysis while the pillars are wet. ${ }^{[15]}$ For CNT forests, Futuba et al. reported a $20 \%$ increase in the packing density as the CNTs were submerged, and significantly more densification during drying. ${ }^{[3]}$

Process Limits and Instabilities: Intricate and uniform filament aggregates are typically representative of a small window of the overall parameter space. Just as particular conditions must be met for assembled structures to be stable,
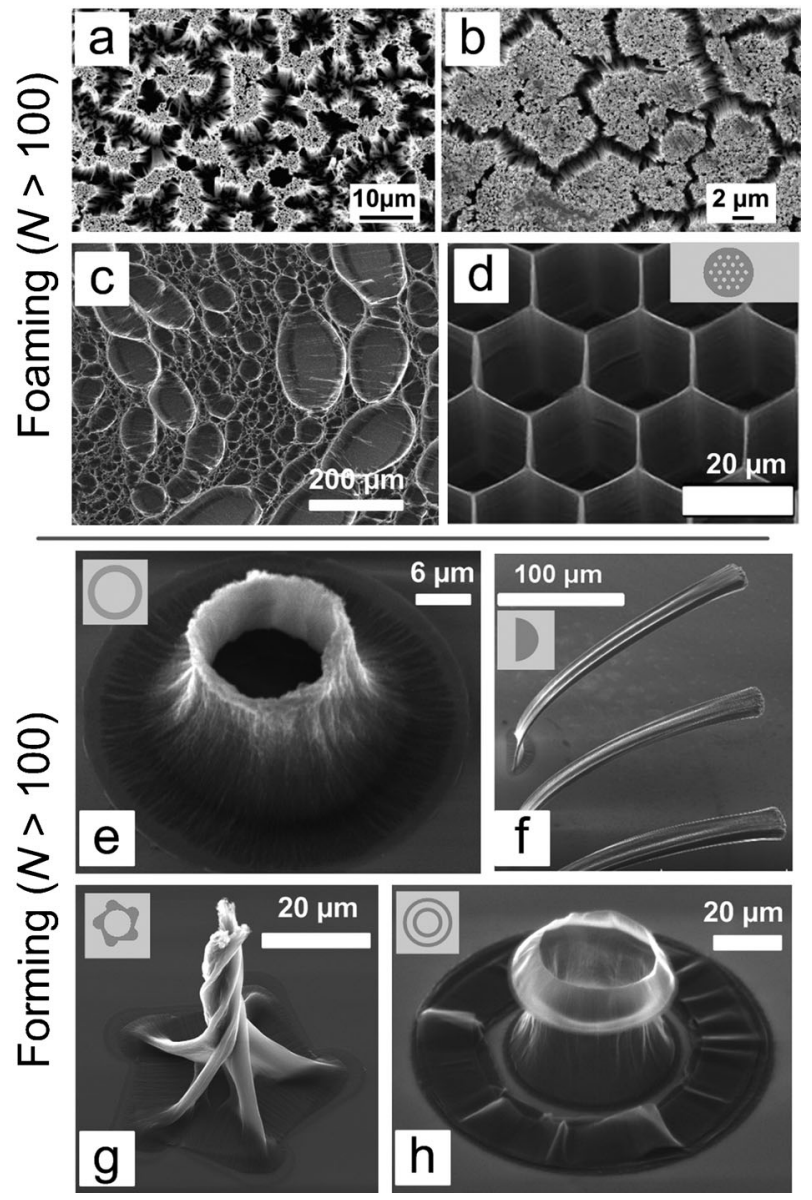

Figure 7. Examples of elastocapillary self-assembly through foaming and forming a) Elastocapillary foaming of a Si nanowire forest. b) Elastocapillary foaming of a Cu nanowire forest. Reprinted from Ref. [38] with permission. Copyright 2009 American Chemical Society. c) Foaming of unpatterned CNT forests using immersion densification d) Templated foaming of patterned CNT forests using condensation densification. Reprinted from Ref. [24] with permission. Copyright 2010 Wiley. e) Forming a cylindrical CNT forest into a cone (initial crosssection outlined in inset). ${ }^{[3]} \mathrm{f}$ ) Capillary forming of CNT forests into bending pillars by condensation. Reprinted from Ref. [73] with permission. Copyright 2011 IEEE. g) Capillary forming of helical CNT micropillars by condensation. Fabrication method similar to that in Ref. [77] h) Fabrication of re-entrant structures by iterating capillary forming and CNT growth; fabrication method similar to that in Ref. [33].

dramatically different outcomes result when, for example, the forces exerted by the liquid exceed the adhesion strength of the filaments to the substrate, or cause the filaments to fold to the substrate rather than remain as stable vertical aggregates. Understanding of these influences can be guided by theory, but is also often quite empirical.

For example, the simple aggregation theory described previously can be used to predict if CNT micropillars will exhibit internal voids after capillary self-assembly, or densify into single solid aggregates (Figure 9a). ${ }^{[19]}$ At the other limit of high aspect ratio structures, such as tall, slender, CNT micropillars or thin, patterned walls, the external capillary forces exerted by the wetting meniscus cause the CNTs to fold to the substrate. ${ }^{[26]}$ Figure $9 \mathrm{~b}$ illustrates how the elastocapillary aggragation of CNT forests transitions from foaming to 
a
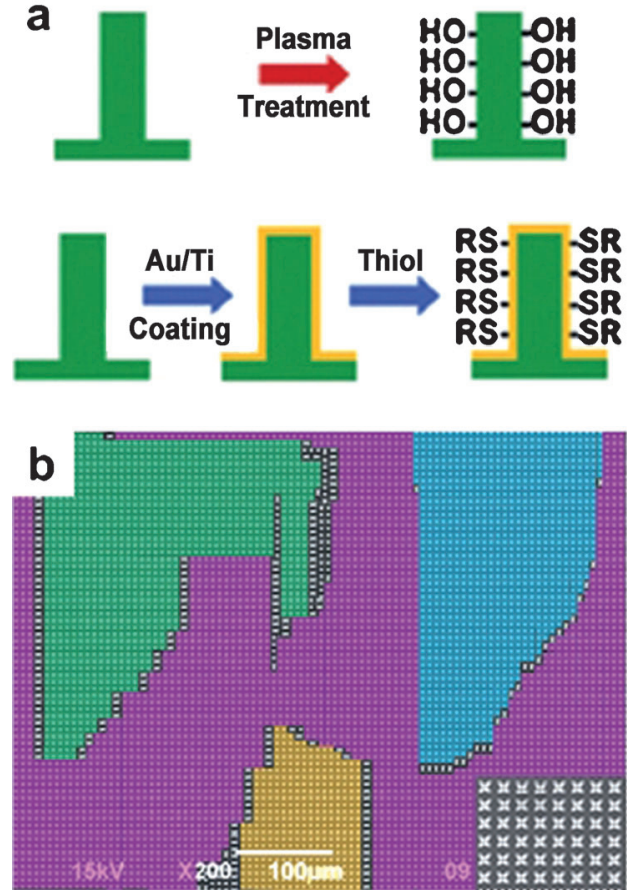

Figure 8. a) Illustration of methods to alter the surface chemistry of nanofilaments. b) Phase diagram of single domain regions within elastocapillary aggregates of polymer nanofilaments. Reprinted from Ref. [16] with permission. Copyright 2012 Elsevier.

forming and folding as a function of the initial forest geometry.

Influence of External Constraints: The aggregation pattern can be influenced by directing the flow of liquid by using an external template. This has been demonstrated using honeycomb structures to confine the densification of polymer nanopillars in smaller compartments and guide the capillary self-assembly into periodic patterns over large areas ${ }^{[16]}$ (Figure $10 \mathrm{a}$ ). It was also observed in this study that the capillary self-assembly of polymer nanopillar arrays facing each other can result in the formation of periodic chiral structures (Figure 10b). ${ }^{[80]}$

Unpublished results on the densification of CNT forests have shown that it is possible to limit the elastocapillary aggregation to, for example, only the bottom part of a CNT forest by applying minute amounts of a densifying liquid (Figure 10c). This enables the fabrication of re-entrant structures with a gradient in mechanical properties. Futhermore, by starting from CNT forests anisotropically coated on only one side by inclined metal evaporation, the elastocapillary aggregation can result in anisotropic pillar movement, including bending pillars as shown in Figure $10 \mathrm{~d}^{[28]}$ This approach enables fine control of the bending angle and the horizontal folding of the pillars.

\section{G. Applications}

In this section we review selected applications of surfaces and devices whose functionality is enhanced by using elastocapillary self-assembly as a fabrication step. Many of a)
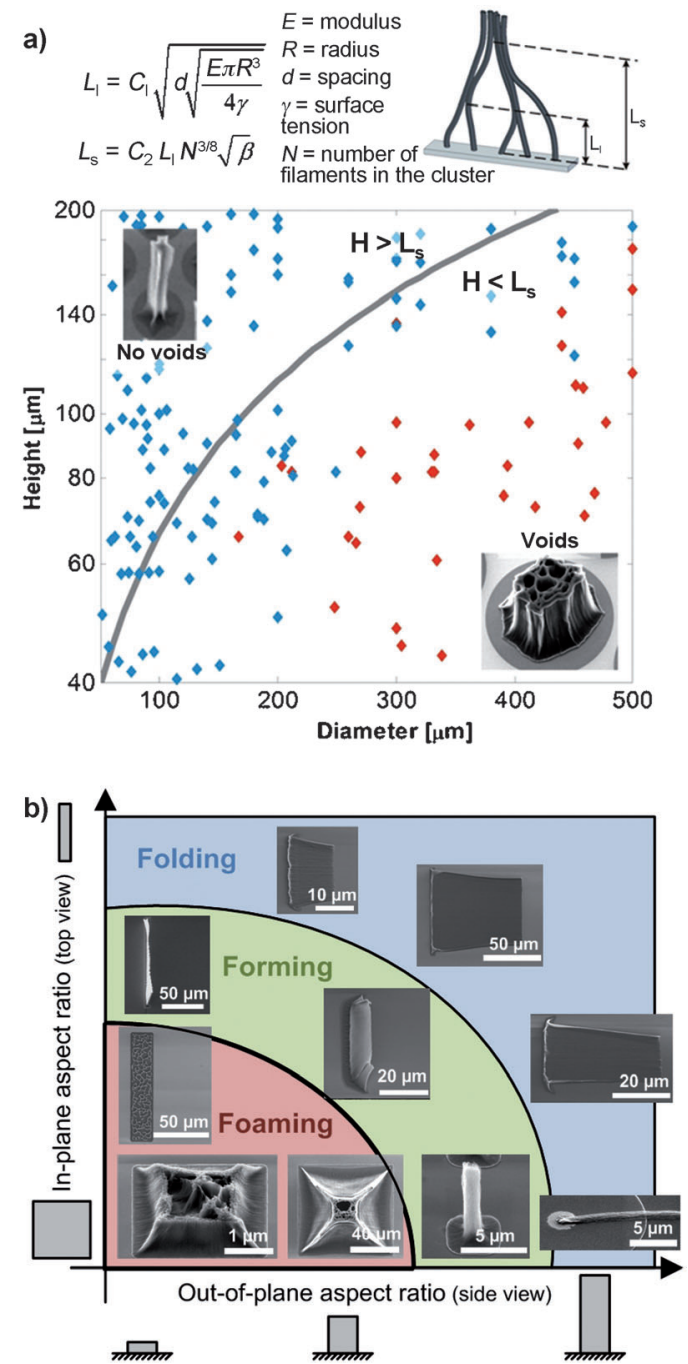

Figure 9. Process limitations in the elastocapillary self-assembly of CNT forests. a) Theoretical and experimental study of the transition between foaming and forming of isolated CNT forest micropillars as a function of height and diameter; adapted from Ref. [19]. b) Picture showing the transition between foaming, forming, and folding in relation to the in-plane and out-of-plane aspect ratio of the CNT micropillars.

these applications use CNTs because of their attractive properties and wide study (Figure 11). However, as methods expand for the fabrication and modification of other nanoscale filaments, this space will undoubtedly broaden in the future (Figure 12).

Microelectronic Vias and Interconnects: The high current carrying capacity of CNTs, which reaches up to $10^{9} \mathrm{Acm}^{-2}$, suggests they could replace $\mathrm{Cu}$ in microelectronic interconnects. ${ }^{[81]}$ The best established method for integrating CNTs into microelectronics is direct growth by CVD ${ }^{[61,62]}$ However, this process often yields an insufficient low packing density of 1 to $5 \% .^{[68]}$ Specifically, the highest density of as-grown SWNTs achieved so far is a factor 30-times lower than this target. ${ }^{[7]}$

Therefore, capillary self-assembly is an attractive means to manipulate low-density CNT forests into densified aggregates with higher conductivity. ${ }^{[17,82]}$ Liu et al. ${ }^{[17]}$ performed an 

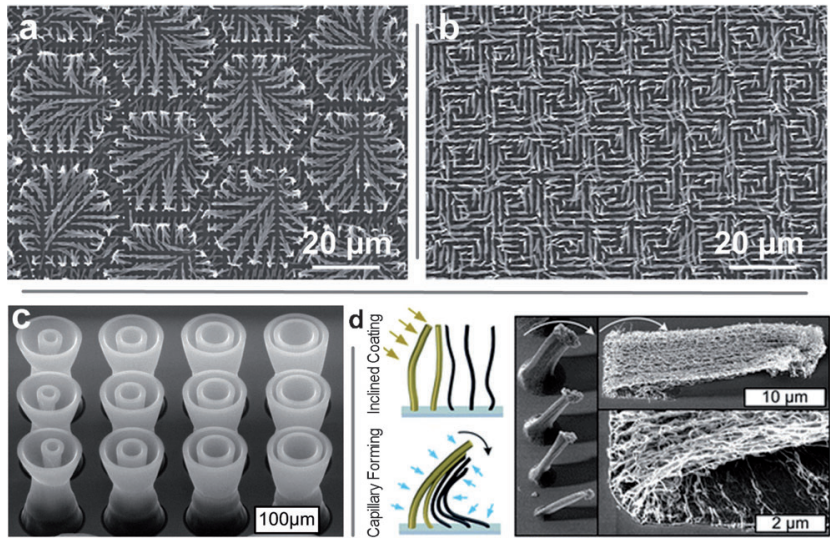

Figure 10. Illustration of methods to guide the elastocapillary process. a) Aggregation templated by a hexagonal lattice. Reprinted from Ref. [16] with permission. Copyright 2012 Elsevier. b) Nanofilaments self-organized into a Moiré pattern by evaporating a liquid sandwiched between two periodic fibrous surfaces. ${ }^{[16]} \mathrm{c}$ ) Partially aggregated CNT forests. d) Capillary bending of Janus CNT forests. Reprinted from Ref. [28] with permission. Copyright 2012 Royal Society of Chemistry.

in-depth investigation of the electrical conductivity of CNT pillars before and after capillary self-assembly, and showed that self-assembly reduced the resistivity 15 -fold through the reduced cross-sectional area. Lim et al. ${ }^{[65]}$ measured a 3.5-fold decrease in resistivity after densification. Importantly, capillary densification of CNT micropillars grown on TiN electrodes also reduced the contact resistance between the CNTs and TiN, by folding the base of the pillar against the substrate while keeping the upper portion vertical. ${ }^{[19]}$

MEMS Devices and Microsensors: Capillary self-assembly is an attractive way to build functional mechanical components by using densely packed nanowires and nanotubes with controlled orientations. For example, Hayamizu et al. demonstrated nanomechanical devices based on horizontally aligned CNT films formed by capillary self-assembly through directed immersion of patterned vertical CNTs. ${ }^{[27]}$ For example, SWNT beams with cross-sections as small as $40 \times 70 \mathrm{~nm}$ (width $\times$ height) were patterned by electron-beam lithography. Each beam consisted of only about 200 SWNTs, and arrays of 1276 electrostatically actuated relay devices were fabricated with a yield of $95.5 \%$ (Figure $11 \mathrm{a}$ ). The same research group also reported the fabrication of freestanding closely packed CNT beams as mechanical resonators. ${ }^{[2]}$

CNT forests were capillary formed into 3D bridges that were infiltrated with hydrogels and integrated into microchannels to form hygroscopic sensors (Figure 11b). ${ }^{[3,84]}$ Vertical CNT sheets were also transformed and densified by capillary folding to connect pairs of patterned electrodes on the substrate. The horizontally aligned CNTs then facilitated directed crystallization of $\mathrm{C}_{60}$ from solution, thus giving the combined hierarchical structure high sensitivity to UV illumination. ${ }^{[70]} \mathrm{CNT}$ "micro-bellows" made by iteration of CNT growth and capillary forming to create novel corrugated structures were shown to have tunable mechanical compliance, which may be useful for compact vertical microsprings in probe card arrays (Figure 11c). By combining top-down lithography and bottom-up self-assembly, Choi et al. devel- oped triangular nanochannels with a width of $30 \mathrm{~nm}$ and a length of $10 \mu \mathrm{m}$ by capillary collapse of electron-beamdefined resist walls (Figure 12b). ${ }^{[4]}$

Trapping Devices and Plasmonic Sensors: Segawa et al. observed that top-gathering pillars can trap and organize piles of particles when the solution contains, for example, $\mathrm{SiO}_{2}$ and $\mathrm{ZnO}$ nanobeads (see Figure $12 \mathrm{a}$ ). The trapped $\mathrm{ZnO}$ particles showed strong photoluminescence. It was found that the topgathering arrays are useful for producing new periodic patterns for luminescence devices. ${ }^{[55-89]}$ In this case, the trapped particles are much smaller than the top-gathering pillars. Later on, Pokroy et al. ${ }^{[55]}$ and Chen et al. ${ }^{[46]}$ showed that beads much larger than individual filaments can be trapped. The authors suggested that controlled particle release from nanopillar aggregates on surfaces could be useful for drug delivery.

On a much smaller scale, Hu et al. demonstrated a molecular trap based on top-gathering pillars. Upon aggregation, the gold-coated nanofilaments formed hot spots for surfaceenhanced Raman spectroscopy (SERS) and X-ray photoelectron spectroscopy (XPS). This approach was used to trap and identify trans-1,2-bis(4-pyridyl)ethylene (Figure $12 \mathrm{c}){ }^{[71,90]}$ Plasmonic structures for SERS were also developed by transferring metal nanoparticles assembled by elastocapillary self-assembly, as shown in Figure 12 e. ${ }^{[71,91]}$ An advantage of this process is the trapping of molecules in between the nanoparticles, and their assembly in the vicinity (smaller than what can be made by lithography).

Bioprobes and Scaffolds: Compared to conventional flat metal electrodes, carbon nanotubes and nanofibers (CNFs) provide a significant increase in surface area as well as good electrochemical stability. As these probes operate in wet environments, the consideration of the use of capillary selfassembly is important. Nguyen- $\mathrm{Vu}$ et al. investigated the influence of capillary self-assembly on CNFs for neural microelectrode arrays. ${ }^{[12]}$ They observed that tips of topgathering CNF microbundles can impale the cell body or push the cell membrane up, similar to a tent pole. Some cell bodies span over the open space and are suspended between the tip of neighboring microbundles, while others lose contact with the neighboring cells and drop into the valley. This topgathering topography yields a very different cell-adhesion mechanism than similar CNF tubes coated with polypyrole (PPy), which did not show capillary self-assembly because of enhanced mechanical stiffness. On a longer term, the elastocapillary self-assembly of nanofilaments could enable extracellular matrices for tissue engineering to be engineered (Figure 11 d). ${ }^{[13,92]}$

Energy: The use of capillary self-assembly to closely pack nanofilaments is useful for supercapacitor electrodes, because of the need for a high-specific surface area. ${ }^{[3]}$ Futuba et al. built two-electrode electric-double-layer capacitor (EDLC) cells out of dense aligned CNT forests, which provided a specific capacitance of $80 \mathrm{Fg}^{-1}$ for a three-electrode cell. ${ }^{[3]}$ Recently, the same research group made thin-film SWCNT capacitors by combining mechanical shearing with capillary self-assembly, thereby resulting in $160 \mathrm{Fg}^{-1}$ (Figure 11e). ${ }^{[2]}$

Pint et al. used capillary self-assembly to fabricate antireflective surfaces for photoelectrochemical devices. ${ }^{[6]} \mathrm{CNF}$ 
a)

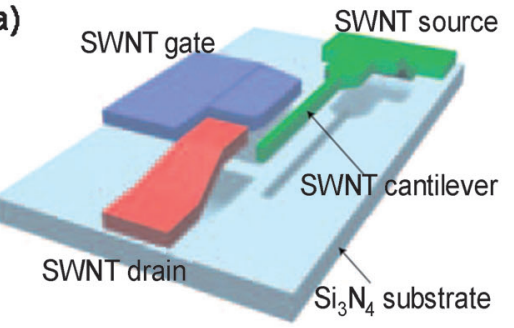

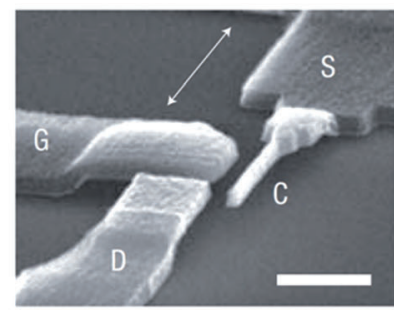

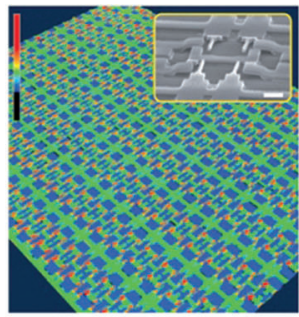

b)
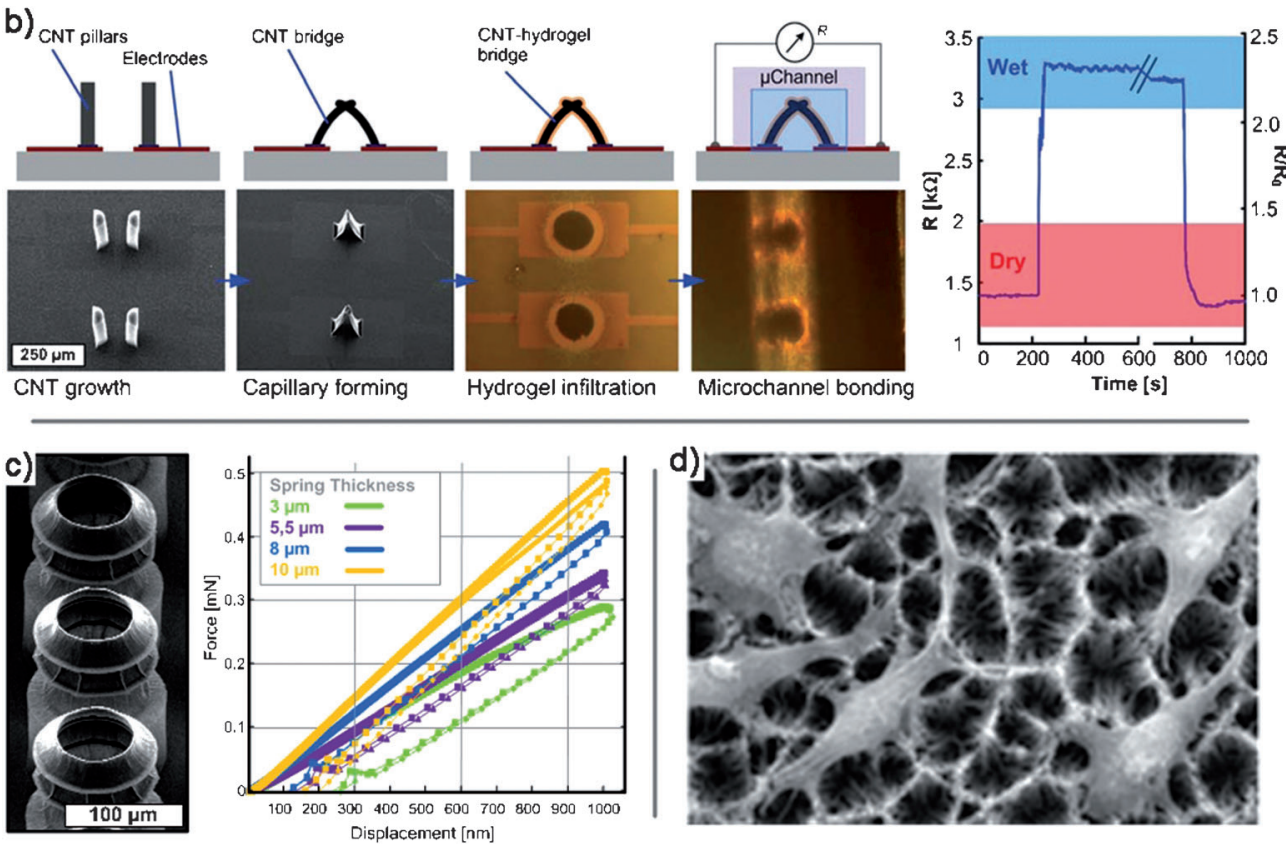

e)
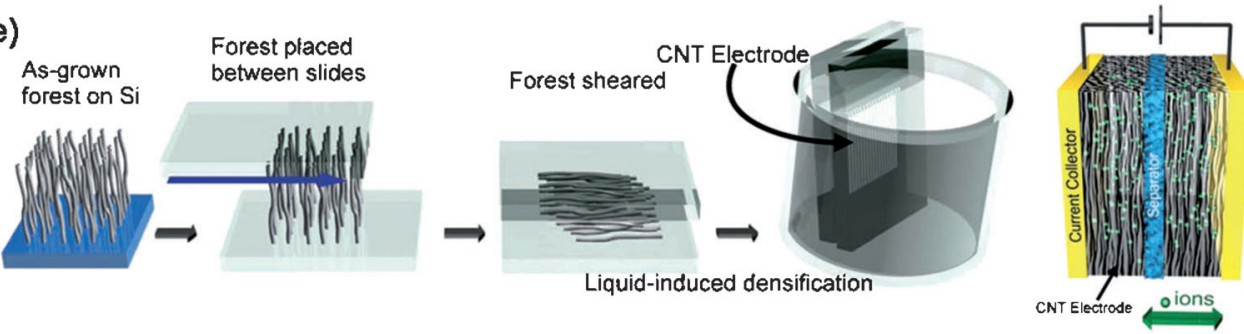

Figure 11. Applications of elastocapillary self-assembled CNTs. a) Microrelays fabricated from horizontal CNTs made by directed immersion aggregation. Reprinted from Ref. [27] with permission. Copyright 2008 Macmillan. b) Hygroscopic microsensors fabricated by capillary forming of 3D CNT-hydrogel sensing nodes. Reprinted from Ref. [83] with permission. Copyright 2011 Royal Chemical Society. c) Corrugated CNT micro-bellows fabricated by iterated CNT growth and capillary forming. Reprinted from Ref. [33] with permission. Copyright 2011 American Chemical Society. d) Foaming carbon nanotube cells for cell growth. Reprinted from Ref. [13] with permission. Copyright 2004 American Chemical Society. e) Supercapacitor fabricated by shearing and immersion densification of CNT forests. Reprinted from Ref. [2] with permission. Copyright 2010 Wiley. compared to unstructured substrates. ${ }^{[38]}$ The foamlike microcavities of $1-6 \mu \mathrm{m}$ width were suggested to nucleate the formation of bubbles during boiling (Figure $7 \mathrm{a}, \mathrm{b})$. Enhanced pool boiling experiments were also conducted with CNT forests, although it is unclear if the nanofilaments were subjected to a capillary self-assembly step prior to boiling. ${ }^{[93]}$

Finally, other researchers suggested the use of elastocapillary aggregation to modulate the color of Bragg diffraction in hydrogel micropillar arrays (Figure $12 \mathrm{~d}),{ }^{[1,42]}$ and to use self-assembled CNT foams as shock-absorbent structural reinforcements, ${ }^{[23]}$ elastic membranes, ${ }^{[23]}$ filtration devices, ${ }^{[23,94]}$ brush motors, ${ }^{[69]}$ temperature sensors, ${ }^{[34]}$ and complex master molds. ${ }^{[20,35]}$

\section{Summary and Outlook}

Elastocapillary aggregation of micro- and nanoscale filaments occurs both in natural and synthetic materials, and the behavior of filaments in liquids is important both for their processing and their use in a variety of applications. While elastocapillary aggregation was initially seen as a nuisance to patterning small flexible features on silicon wafers, it is now arrays were top-gathered by condensation and then coated with $\mathrm{TiO}_{2}$ layers by atomic layer deposition (ALD). They demonstrated an up to threefold enhancement of the shortcircuit current density in the capillary-texturized $\mathrm{CNF} / \mathrm{TiO}$ photoelectrochemical device compared to a planar equivalent.

The hierarchical and robust surface textures made by capillary self-assembly can be useful for engineering a phasechange heat transfer. Capillary self-assembled nanowire arrays of $\mathrm{Si}$ and $\mathrm{Cu}$ showed higher heat transfer coefficients and increased the critical heat flux by more than $100 \%$ being embraced as a means of fabricating complex hierarchical surface structures. Importantly, this process is inherently cost-effective and scalable, and takes advantage of wellknown planar patterning methods to create new geometries that cannot be made by other methods. Recent studies show that hierarchical aggregates of nanofilaments are particularly promising for creating high-surface-area materials for energy applications as well as for fabricating surface texture with controllable wetting, adhesion, optic, or electrical properties. Further developments in the fabrication of nanofilaments will extend the versatility of elastocapillary self-assembly beyond 

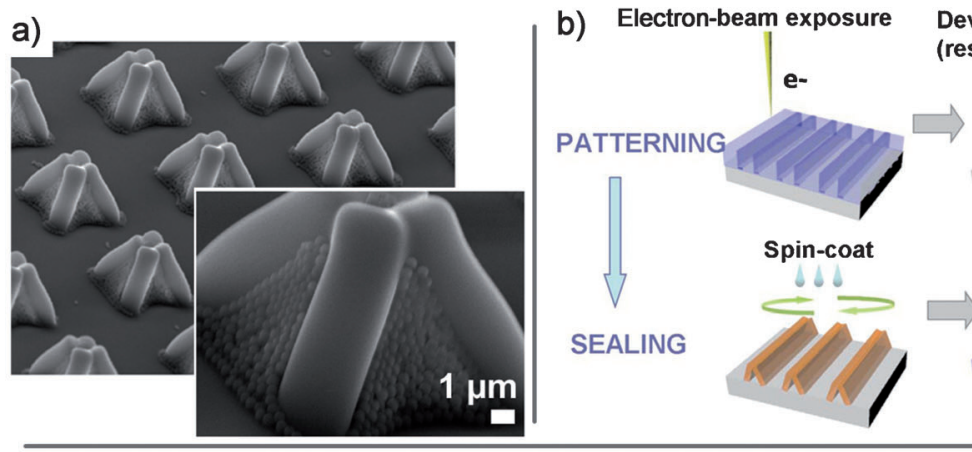

evelop, rinse, and dry sist collapse)
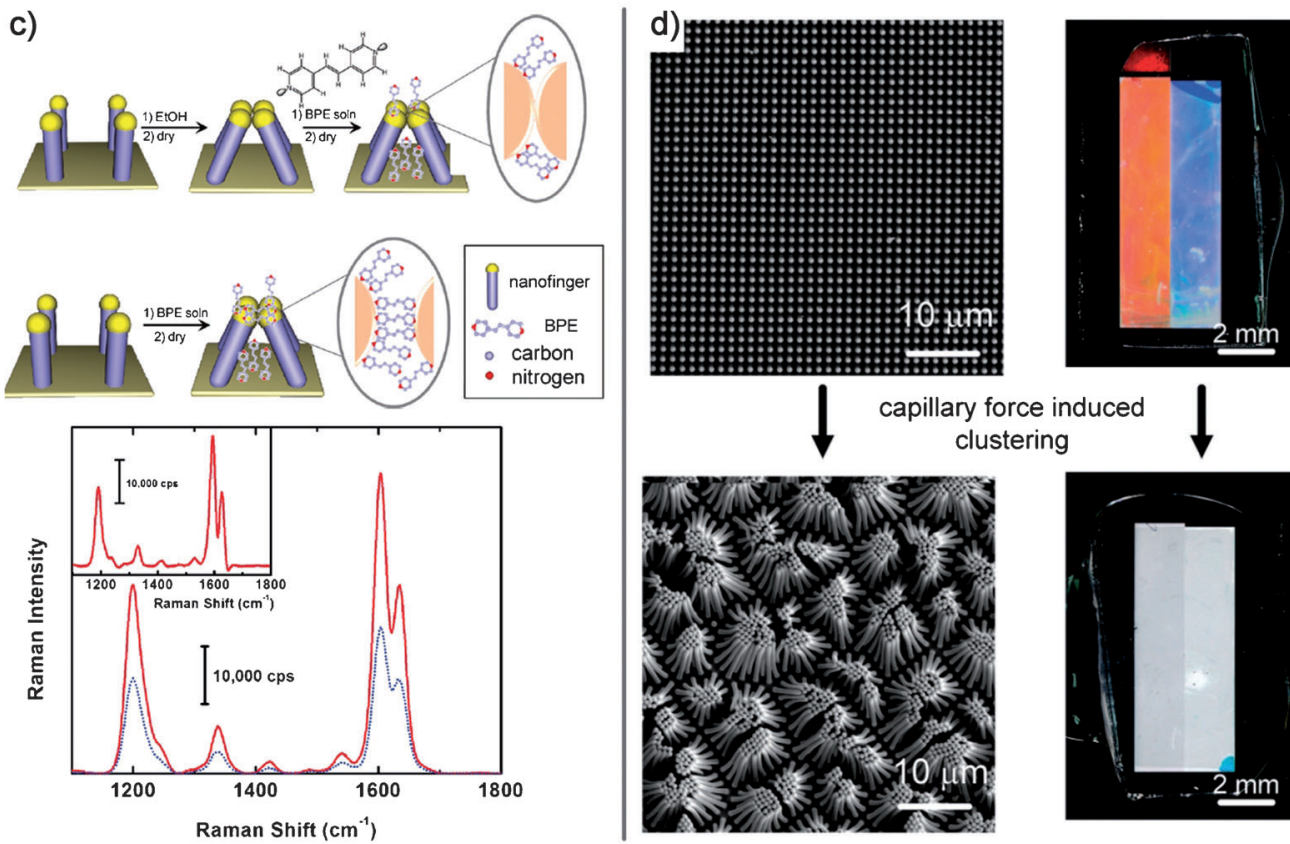

Spin-coat

SEALING
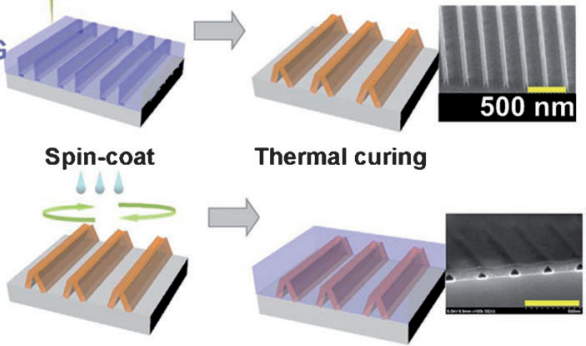

Thermal curing

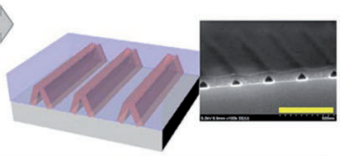

\section{.} Tawfick for helpful discussions and contributions to Figure 9.

Received: July 25, 2012

Published online: January 21, 2013

[1] D. Chandra, S. Yang, Acc. Chem. Res. 2010, 43, 1080-1091.

[2] A. Izadi-Najafabadi, S. Yasuda, K. Kobashi, T. Yamada, D. N. Futaba, H. Hatori, M. Yumura, S. Iijima, K. Hata, $A d v$. Mater. 2010, 22, E235E241.

[3] D. N. Futaba, K. Hata, T. Yamada, T. Hiraoka, Y. Hayamizu, Y. Kakudate, O. Tanaike, H. Hatori, M. Yumura, S. Iijima, Nat. Mater. 2006, 5, 987-994.

e)

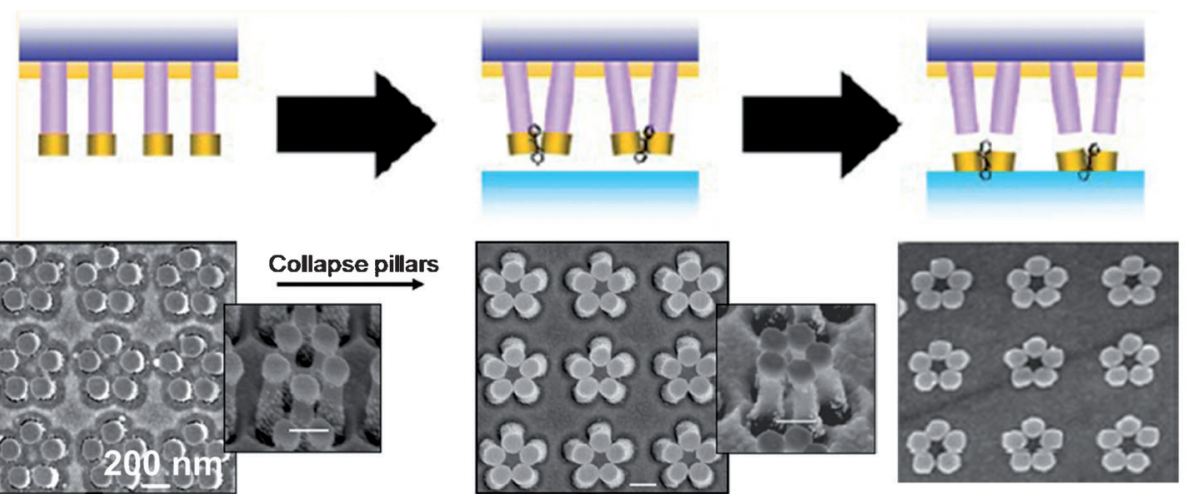

Figure 12. Applications of elastocapillary-aggregated polymer nanofilaments. a) Trapping of nanoparticles under top-gathering pillars. Reprinted from Ref. [87] with permission. Copyright 2008 Elsevier. b) Fabrication of nanoscale 3D channels by aggregation of electron-beam nanosheets. Reprinted from Ref. [47] with permission. Copyright 2008 American Institute of Physics. c) Trapping of molecules in top-gathering pillars, and comparison of Raman spectra with trapped molecules (the inset shows the contribution from the molecules trapped in the fingertips). Reprinted from Ref. [71] with permission. Copyright 2011 American Chemical Society. d) Elastocapillary modulation of the Bragg diffraction color in hydrogel filaments. Reprinted from Ref. [42] with permission. Copyright 2009 American Chemical Society. e) Trapping of molecules in metal nanoparticles and transfer printing thereof. Reprinted from Ref. [91] with permission. Copyright 2012 American Chemical Society.
[4] H. J. Gao, X. Wang, H. M. Yao, S. Gorb, E. Arzt, Mech. Mater. 2005, $37,275-285$.

[5] L. Qu, L. Dai, M. Stone, Z. Xia, Z. L. Wang, Science 2008, 322, 238-242.

[6] C. L. Pint, K. Takei, R. Kapadia, M. Zheng, A. C. Ford, J. Zhang, A. Jamshidi, R. Bardhan, J. J. Urban, M. Wu, J. W. Ager, M. M. Oye, A. Javey, $A d v$. Energy Mater. 2011, 1, 10401045.

[7] G. K. Mor, K. Shankar, M. Paulose, O. K. Varghese, C. A. Grimes, Nano Lett. 2006, 6, 215 218.

[8] K. K. S. Lau, J. Bico, K. B. K. Teo, M. Chhowalla, G. A. J. Amarathe most common materials used today, and will lead to industrial implementation for large-area nanomanufacturing. tunga, W. I. Milne, G. H. McKinley, K. K. Gleason, Nano Lett. 2003, 3, 1701-1705.

[9] N. Mingo, D. A. Broido, Phys. Rev. Lett. 2005, 95, 096105. 
[10] N. Kaji, Y. Tezuka, Y. Takamura, M. Ueda, T. Nishimoto, H. Nakanishi, Y. Horiike, Y. Baba, Anal. Chem. 2004, 76, 15-22.

[11] F. Fachin, G. D. Chen, M. Toner, B. L. Wardle, J. Microelectromech. Syst. 2011, 20, 1428-1438.

[12] T. D. B. Nguyen-Vu, H. Chen, A. M. Cassell, R. Andrews, M. Meyyappan, J. Li, Small 2006, 2, 89-94.

[13] M. A. Correa-Duarte, N. Wagner, J. Rojas-Chapana, C. Morsczeck, M. Thie, M. Giersig, Nano Lett. 2004, 4, 2233-2236.

[14] S. Tawfick, M. De Volder, D. Copic, S. J. Park, C. R. Oliver, E. S. Polsen, M. J. Roberts, A. J. Hart, Adv. Mater. 2012, 24, 1628 1674.

[15] T. Tanaka, M. Morigami, N. Atoda, Jpn. J. Appl. Phys. Part 1 1993, 32, 6059-6064.

[16] A. Grinthal, S. H. Kang, A. K. Epstein, M. Aizenberg, M. Khan, J. Aizenberg, Nano Today 2012, 7, 35-52.

[17] Z. C. Liu, L. J. Ci, S. Kar, P. M. Ajayan, J. Q. Lu, IEEE Trans. Nanotechnol. 2009, 8, 196-203.

[18] E. Verploegen, A. J. Hart, M. De Volder, S. Tawfick, K. K. Chia, R. E. Cohen, J. Appl. Phys. 2011, 109, 094316.

[19] M. F. L. De Volder, S. J. Park, S. H. Tawfick, D. O. Vidaud, A. J. Hart, J. Micromech. Microeng. 2011, 21, 045033.

[20] D. Copic, S. J. Park, S. Tawfick, M. F. L. De Volder, A. J. Hart, Lab Chip 2011, 11, 1831-1837.

[21] C. Journet, S. Moulinet, C. Ybert, S. T. Purcell, L. Bocquet, Europhys. Lett. 2005, 71, 104-109.

[22] D. Copic, S. J. Park, S. Tawfick, M. De Volder, A. J. Hart, J. Visualized Exp. 2012, 65, e3980.

[23] N. Chakrapani, B. Q. Wei, A. Carrillo, P. M. Ajayan, R. S. Kane, Proc. Natl. Acad. Sci. USA 2004, 101, 4009-4012.

[24] M. De Volder, S. H. Tawfick, S. J. Park, D. Copic, Z. Zhao, W. Lu, A. J. Hart, Adv. Mater. 2010, 22, 4384-4389.

[25] H. Liu, S. H. Li, J. Zhai, H. J. Li, Q. S. Zheng, L. Jiang, D. B. Zhu, Angew. Chem. 2004, 116, 1166-1169; Angew. Chem. Int. Ed. 2004, 43, 1146-1149.

[26] S. Tawfick, M. De Volder, A. J. Hart, Langmuir 2011, 27, 63896394.

[27] Y. Hayamizu, T. Yamada, K. Mizuno, R. C. Davis, D. N. Futaba, M. Yumura, K. Hata, Nat. Nanotechnol. 2008, 3, 289-294.

[28] S. Tawfick, A. J. Hart, M. De Volder, Nanoscale 2012, 4, 3852 3856.

[29] Y. Hayamizu, R. C. Davis, T. Yamada, D. N. Futaba, S. Yasuda, M. Yumura, K. Hata, Phys. Rev. Lett. 2009, 102, 175505.

[30] E. R. Meshot, K. D. Patel, S. Tawfick, K. A. Juggernauth, M. Bedewy, E. A. Verploegen, M. F. L. De Volder, A. J. Hart, $A d v$. Funct. Mater. 2012, 22, 577-584.

[31] M. F. L. De Volder, D. O. Vidaud, E. R. Meshot, S. Tawfick, A. J. Hart, Microelectron. Eng. 2010, 87, 1233-1238.

[32] See Ref. [24].

[33] M. F. L. De Volder, S. Tawfick, S. J. Park, A. J. Hart, ACS Nano 2011, 5, 7310-7317.

[34] M. De Volder, D. Reynaerts, C. Van Hoof, S. Tawfick, A. J. Hart, IEEE Sensors 2010, 2369-2372.

[35] see Ref. [22].

[36] H. Cai, Y. Xu, P. G. He, Y. Z. Fang, Electroanalysis 2003, 15 , $1864-1870$

[37] Y. P. Zhao, J. G. Fan, Appl. Phys. Lett. 2006, 88, 103123.

[38] R. Chen, M.-C. Lu, V. Srinivasan, Z. Wang, H. H. Cho, A. Majumdar, Nano Lett. 2009, 9, 548-553.

[39] H. Namatsu, K. Kurihara, M. Nagase, K. Iwadate, K. Murase, Appl. Phys. Lett. 1995, 66, 2655-2657.

[40] J. J. Hill, K. Haller, B. Gelfand, K. J. Ziegler, ACS Appl. Mater. Interfaces 2010, 2, 1992-1998.

[41] A. Dev, S. Chaudhuri, Nanotechnology 2007, 18, 175607.

[42] D. Chandra, S. Yang, A. A. Soshinsky, R. J. Gambogi, ACS Appl. Mater. Interfaces 2009, 1, 1698-1704.

[43] H. Duan, K. K. Berggren, Nano Lett. 2010, 10, 3710-3716.
[44] H. Duan, J. K. W. Yang, K. K. Berggren, Small 2011, 7, 2661 2668.

[45] A. Ishikawa, M. Sakata, A. Kawai, J. Photopolym. Sci. Technol. 2004, 17, 457-460.

[46] B. Chen, S. Seidel, H. Hori, M. Gupta, ACS Appl. Mater. Interfaces 2011, 3, 4201-4205.

[47] S. Choi, M. Yan, I. Adesida, Appl. Phys. Lett. 2008, 93, 163113.

[48] D. E. Noga, R. A. Lawson, C.-T. Lee, L. M. Tolbert, C. L. Henderson in Conference on Advances in Resist Materials and Processing Technology XXVI, San Jose, CA, 2009.

[49] J. Bico, B. Roman, L. Moulin, A. Boudaoud, Nature 2004, 432, $690-690$

[50] M. Mastrangeli, S. Abbasi, C. Varel, C. Van Hoof, J. P. Celis, K. F. Boehringer, J. Micromech. Microeng. 2009, 19, 045015.

[51] B. Roman, J. Bico, J. Phys. Condens. Matter 2010, 22, 493101.

[52] M. Grzelczak, J. Vermant, E. M. Furst, L. M. Liz-Marzan, ACS Nano 2010, 4, 3591-3605.

[53] G. M. Whitesides, B. Grzybowski, Science 2002, 295, 2418-2421.

[54] N. Vogel, C. K. Weiss, K. Landfester, Soft Matter 2012, 8, $4044-$ 4061.

[55] B. Pokroy, S. H. Kang, L. Mahadevan, J. Aizenberg, Science 2009, 323, 237-240.

[56] S. H. Kang, B. Pokroy, L. Mahadevan, J. Aizenberg, Acs Nano 2010, 4, 6323-6331.

[57] M. Matsunaga, M. Aizenberg, J. Aizenberg, J. Am. Chem. Soc. 2011, 133, 5545-5553.

[58] P. Kim, W. E. Adorno-Martinez, M. Khan, J. Aizenberg, Nat. Protoc. 2012, 7, 311-327.

[59] M. F. L. De Volder, R. Vansweevelt, P. Wagner, D. Reynaerts, C. Van Hoof, A. J. Hart, Acs Nano 2011, 5, 6593-6600.

[60] C. L. Cheung, R. J. Nikolic, C. E. Reinhardt, T. F. Wang, Nanotechnology 2006, 17, 1339-1343.

[61] A. J. Hart, A. H. Slocum, J. Phys. Chem. B 2006, 110, 82508257.

[62] K. Hata, D. N. Futaba, K. Mizuno, T. Namai, M. Yumura, S. Iijima, Science 2004, 306, 1362-1364.

[63] See Ref. [31]

[64] See Ref. [25].

[65] X. D. Lim, H. W. G. Foo, G. H. Chia, C. H. Sow, ACS Nano 2010 , 4, 1067-1075.

[66] J. Liang, R. G. D. Jeyasingh, H.-Y. Chen, H. S. P. Wong, IEEE Trans. Electron Devices 2012, 59, 1155-1163.

[67] M. De Volder, J. Decoster, D. Reynaerts, C. Van Hoof, S.-G. Kim, Small 2012, 8, 2006.

[68] Z. Liu, N. Bajwa, L. Ci, S. H. Lee, S. Kar, P. M. Ajayan, et al., 10th International Interconnect Technology Conference 2007, pp. $201-203$.

[69] D. N. Futaba, K. Miyake, K. Murata, Y. Hayamizu, T. Yamada, S. Sasaki, M. Yumura, K. Hata, Nano Lett. 2009, 9, 3302-3307.

[70] See Ref.[30].

[71] A. Kim, F. S. Ou, D. A. A. Ohlberg, M. Hu, R. S. Williams, Z. Li, J. Am. Chem. Soc. 2011, 133, 8234-8239.

[72] G. Liu, J. Zhou, Y. Xiong, X. Zhang, Y. Tian, Nanotechnology 2011, 22, 305305 .

[73] M. De Volder, S. Tawfick, S. J. Park, D. Copic, A. J. Hart, TRANSDUCERS 2011-2011 16th International Solid-State Sensors, Actuators and Microsystems Conference, 01, 2011.

[74] S. Esconjauregui, M. Fouquet, B. C. Bayer, C. Ducati, R. Smajda, S. Hofmann, J. Robertson, ACS Nano 2010, 4, 7431-7436.

[75] D. Wu, Q.-D. Chen, B.-B. Xu, J. Jiao, Y. Xu, H. Xia, H. B. Sun, Appl. Phys. Lett. 2009, 95, 091902.

[76] F. Járai-Szabó, E.-A. Horvat, R. Vajtai, Z. Neda, Chem. Phys. Lett. 2011, 511, 378-383.

[77] See Ref. [22].

[78] Z. Z. Zhao, S. H. Tawfick, S. J. Park, M. De Volder, A. J. Hart, W. Lu, Phys. Rev. E 2010, 82, 041605. 
[79] J. Qu, Z. Zhao, X. Wang, J. Qiu, J. Mater. Chem. 2011, 21, 5967 5971.

[80] S. H. Kang, N. Wu, A. Grinthal, J. Aizenberg, Phys. Rev. Lett. 2011, 107, 177802 .

[81] N. Chiodarelli, Y. Li, D. J. Cott, S. Mertens, N. Peys, M. Heyns, S. D. Gendt, G. Groeseneken, P. M. Vereecken, Microelectron. Eng. 2011, 88, 837-843.

[82] T. Wang, S. Chen, D. Jiang, Y. Fu, K. Jeppson, L. Ye, J. Liu, IEEE Electron Device Lett. 2012, 33, 420-422.

[83] M. De Volder, S. H. Tawfick, D. Copic, A. J. Hart, Soft Matter 2011, 7, $9844-9847$.

[84] M. De Volder, S. Tawfick, D. Copic, A. J. Hart, TRANSDUCERS 2011-2011 16th International Solid-State Sensors, Actuators and Microsystems Conference, 012011.

[85] H. Segawa, S. Yamaguchi, Y. Yamazaki, T. Yano, S. Shibata, H. Misawa, Appl. Phys. A 2006, 83, 447-451.

[86] H. Segawa, Y. Yamazaki, T. Yano, S. Shibata, Mater. Sci. Eng. B 2008, $148,43-47$.
[87] H. Segawa, Y. Yamazaki, S. Tachiki, T. Yano, S. Shibata, J. Colloid Interface Sci. 2008, 323, 187-190.

[88] H. Segawa, S. Tachiki, T. Yano, S. Shibata, Mater. Sci. Eng. B 2009, 161, 85-90.

[89] H. Segawa, D. Morishima, T. Yano, S. Shibata, Mater. Sci. Eng. B 2010, 173, $162-167$.

[90] M. Hu, F. S. Ou, W. Wu, I. Naumov, X. Li, A. M. Bratkovsky, R. S. Williams, Z. Y. Li, J. Am. Chem. Soc. 2010, 132, 1282012822.

[91] S. Barcelo, A. Kim, W. Wu, Z. Li, ACS Nano 2012, 6, 6446-6452.

[92] T. Dvir, B. P. Timko, D. S. Kohane, R. Langer, Nat. Nanotechnol. 2011, $6,13-22$.

[93] H. S. Ahn, N. Sinha, M. Zhang, D. Banerjee, S. Fang, R. H. Baughman, J. Heat Transfer 2006, 128, 1335-1342.

[94] M. Yu, H. H. Funke, J. L. Falconer, R. D. Noble, Nano Lett. 2009, 9, 225-229. 